\title{
Congenital Hypercoagulable Disorders
}

\author{
Federico Cacciapuoti*
}

*Department of Internal Medicine, Second University of Naples, Italy

${ }^{\star}$ Correspondence to: Federico Cacciapuoti MD, Second University of Naples-Italy, Piazza L. Miraglia,2, 80138-Naples-Italy; E-mail: federico.cacciapuoti@unina2.it

Received: August 19, 2016; Accepted: September 17, 2016; Published: October 02, 2016

\begin{abstract}
The blood coagulation in the body's vessels represents a main cause of thrombosis arterial and venous acute and chronic events. The causes of hypercoagulation can be acquired or inherited. Acquired hypercoagulable disorders most frequently occurs. It can be present in several conditions, included advanced age, and must be reported to one or more factors of Virchow's triad. On the contrary, hereditary thrombophilia is less frequent and little known too. Its pictures manifest with repeated vascular acute events in subjects in young age, often belonging to the same family, in the absence of factor of Virchow's triad. Inherited hypercoagulation is due to some genetic anomaly of MTHFR, Factor V Leiden, Factor II. Nevertheless, these states can be caused by a deficiency of anticoagulant proteins (Protein C, Protein S, Antithrombin III) or by antiphospholipid antibodies syndrome. But the anticoagulant tendency, besides congenital, may be also dependent by some acquired conditions, such as liver disease, pregnancy, some infection and the use of drugs, as estrogen, heparin or warfarin. The genetic causes and the pathogenetic mechanisms of inherited thrombophilia were briefly illustrated.
\end{abstract}

Key words: Hypercoagulability, hereditary thrombophilia, MTHFR, Factor V Leiden, Prothrombin, Anticoagulant proteins, Lupus Anticoagulant.

\section{Introduction}

Hemostasis is highly regulated by coagulant and anticoagulant factors to maintain a balance between bleeding and coagulation. On the contrary, the prevalence of pro-coagulant on anti-coagulant factors causes an impaired hemostasis balance, inducing thrombosis that blocks or reduces blood supply in tissues. That represents a most frequent and important life-threatening human disease. Hypercoagulable states can be acquired and congenital [1]. Both conditions likely to develop clots in venous and arterial vessels. Venous thrombosis is the most common cause of potentially lifethreatening blood clots in the lungs, the deep leg veins, the arm veins, the kidney veins, or others. Conversely, arterial thrombosis consists in clot-formation in the arteries, that can cause some complications associated with significant morbidity and mortality, such as acute myocardial infarction, stroke or TIA [2].

\section{Acquired Hypercoagulability}

Acquired form can happen in some conditions, as advanced age, diabetes, inflammation, cancer, obesity, immobility, and others. These act favouring one or more factors of Virchow's triad (stasis, endothelial dysfunction, hypercoagulability) causing procoagulant tendency [3] .

\section{Congenital Hypercoagulability}

Also defined hereditary thrombophilia, it occurs lesser than acquired type. It is the result of abnormal gene deriving from one (heterozygous) of both parents (homozygous). It should be suspected in patients with individual and/or familiar history of recurrent ischemic-thrombotic events, in absence of any of the risk factors related to the conditions reported above (Virchow's triad). But, hereditary thrombophilia generally is also present in subjects at a young age (40 years), with history of thrombosis in unusual sites (mesenteric, renal, hepatic, retinal veins) or cerebral thrombosis [4].

Genes mostly codifying for hereditary thrombophilia include the following:

- MTHFR (Methylene-Tetra-Hydro-Folate-Reductase);

- Factor V Leiden (factor V);

- Prothrombin (factor II).

Reduced MTHRF activity may be responsible for increased homocysteine levels (HHcy) that is a risk factor for arterial and venous blood clots. HHcy exerts its thrombotic effect acting both directly on endothelial layer and indirectly through DNA-hypomethylation $[5,6]$. Factor V Leiden (FVL) is factor of hereditary thrombophilia in Caucasian populations. Heterozygous FVL is much more common than homozygous. FVLa causes the activation of prothrombin in thrombin [7]. It predisposes mainly to venous thrombosis (especially retinal vein occlusion). Finally, activated prothrombin induces fibrinogen in to fibrin conversion and favours deep venous thrombosis.

Other conditions causing a hypercoagulable state are induced by the deficiencies (inherited or acquired) of the following proteins defined as natural anticoagulants: Protein C; Protein S; Antithrombin III.

Once the coagulation process begins, these act limiting the process in accordance with the scheme following: (figure. 1)

Activated protein $\mathrm{C}$ and protein $\mathrm{S}$ act by inhibiting the action of the cofactors (factor Va and factor VIIIa). Antithrombin inhibits the serine proteases (factor III, X, XI, XII). Obviously, deficiencies of these proteins are associated with thromboembolic disease [8]. 


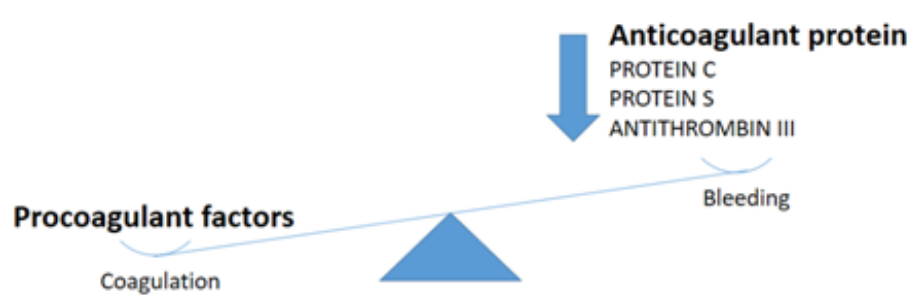

Figure. 1: Prevalence of coagulation on bleeding induced by reduction of anticoagulant proteins

Deficiencies of anticoagulant factors are inherited for patient's parents. People born with deficiencies of one of the abnormal gene from either mother or father is heterozygous for this gene (more frequent). Conversely, patients can inherit abnormal gene from both parents. These are homozygous and rarely occurs. Nevertheless, deficiencies of anticoagulant factors can also be acquired. Individuals with normal levels of anticoagulants may develop deficiencies in certain situations, such as pregnancy, liver disease, some infections or vitamin $\mathrm{K}$ deficiency and the use of certain medication, such as estrogen, heparin, warfarin [9].

Finally, antiphospholipid antibodies syndrome (also called lupus anticoagulant) must be considered such as a cause of inherited thrombophilia. It occurs in about $20 \%$ of patients with systemic lupus erythematous (SLE) and may be also associated with other autoimmune diseases [10]. The inappropriate name for antibodies is due to the initial discovery in patients with SLE, although they can also occur in individuals without lupus. The mechanisms of thrombosis in this syndrome are not yet defined. Nevertheless, activation of platelets to enhance endothelial adherence or production of antibodies against protein C or protein S must be considered [11].

\section{Conclusions}

Arterial and/or thromboembolic events deriving from acquired hypercoagulability represent the most frequent causes of morbidity and mortality of the whole population and were enough studied. On the contrary, disorders deriving from inherited thrombophilia less frequently occur and its physiopathology barely is known. Thus, the better knowledge of its numerous types is requested to successfully contrast its dreadful complications.

\section{References}

1. Kitchens CS (1985) Concept of hypercoagulability: a review of its development, clinical application, and recent progress. Semin Thromb Hemost 11: 293-315. [crossref]

2. Reiner AP, Siscovick DS, Rosendaal FR (2001) Hemostatic risk factors and arterial thrombotic disease. Thromb Haemost 85: 584-595. [crossref]

3. Franchini M, Mannucci PM (2008) The hemostatic balance revised through the lessons of markind evolution. Intern Emerg Med 3: 3-8

4. Khan S, Dickerman JD (2006) Hereditary thrombophilia. Thromb J 4: 15. [crossref]

5. Welch GN, Loscalzo J (1998) Homocysteine and atherothrombosis. $N$ Engl $J$ Med 338: 1042-1050. [crossref]

6. Cacciapuoti F (2011) Hyper-homocysteinemia: a novel risk factor or a powerful marker for cardiovascular diseases? Pathogenetic and therapeutical uncertainties. $J$ Thromb Thrombolysis 32: 82-88. [crossref]

7. De Stefano V, Chiusolo P, Paciaroni K, Leone G (1998) Epidemiology of factor V Leiden: clinical implications. Semin Thromb Hemost 24: 367-379. [crossref]
8. High KA (1988) Antithrombin III, protein C, and protein S. Naturally occurring anticoagulant proteins. Arch Pathol Lab Med 112: 28-36. [crossref]

9. Lipe B, Ornstein DL (2011) Deficiencies of natural anticoagulants, protein C, protein S, and antithrombin. Circulation 124: e365-368. [crossref]

10. Lockshin MD (2008) Update on antiphospholipid syndrome. Bull NYU Hosp Jt Dis 66: 195-197. [crossref]

11. Negrini S, Pappalardo F, Murdaca G, Indiveri F, et al. (2016) The antiphospholipid syndrome: from pathophysiology to treatment. Clin Exp Med . [crossref]
Citation:

Federico Cacciapuoti (2016) Congenital Hypercoagulable Disorders. Int Med Res Open $J$ Volume 1(1): 1-2 